PHYSICAL REVIEW D 92, 054014 (2015)

\title{
Heavy quarkonia in strong magnetic fields
}

\author{
Claudio Bonati" \\ INFN-Sezione di Pisa, Largo Pontecorvo 3, I-56127 Pisa, Italy \\ Massimo D’Elia ${ }^{\dagger}$ \\ INFN_Sezione di Pisa, Largo Pontecorvo 3, I-56127 Pisa, Italy \\ and Dipartimento di Fisica dell'Università di Pisa, Largo Pontecorvo 3, I-56127 Pisa, Italy \\ Andrea Rucci \\ Dipartimento di Fisica dell'Università di Pisa, Largo Pontecorvo 3, I-56127 Pisa, Italy
}

(Received 2 July 2015; published 10 September 2015)

\begin{abstract}
We investigate the influence of a homogeneous and constant strong external magnetic field on the heavymeson spectrum. Quarkonium states $c \bar{c}$ and $b \bar{b}$ are described within a nonrelativistic framework and by means of a suitable potential model based on the Cornell parametrization. In particular, in this work we propose a model which takes into account the possible anisotropies emerging at the level of the static quarkantiquark potential, as observed in recent lattice studies. The investigation is performed both with and without taking into account the anisotropy of the static potential, in order to better clarify its effects.
\end{abstract}

DOI: 10.1103/PhysRevD.92.054014

PACS numbers: 12.39.Jh, 12.38.Gc, 12.39.Pn, 14.40.Pq

\section{INTRODUCTION}

In recent times there has been a great interest regarding the physics of strongly interacting matter in the presence of strong external magnetic fields, i.e. such that ${ }^{1} e B \simeq m_{\pi}^{2}$ or larger (see, e.g., Refs. [1,2] for recent reviews). This topic could be relevant to the study of some dense astrophysical objects, like magnetars [3], and for cosmology [4,5]. However, the main interest was triggered by the fact that magnetic fields of this order of magnitude can be created in a laboratory, in particular in heavy-ion collisions [6-10], when two relativistic heavy ions collide with a nonzero impact parameter, producing a huge field in the collision region. For example, one can reach $|e| B \simeq 0.2-0.3 \mathrm{GeV}^{2}$ in $\mathrm{Pb}+\mathrm{Pb}$ collisions at the Large Hadron Collider (LHC).

Such huge magnetic fields are produced in the very early stages of the collision. It is still not clear how long and to what extent they survive the thermalization process of the fireball created after the collision. Therefore, while various theoretical investigations, based both on model studies and on lattice QCD simulations (LQCD), have predicted many interesting phenomena affecting the properties of strongly interacting matter in the presence of strong magnetic backgrounds, it is still uncertain to what extent such phenomena will be detectable in heavy ion experiments.

In this perspective, effects regarding the physics of heavy flavors are of particular interest, since they are more sensitive to the conditions taking place in the early stages

\footnotetext{
*claudio.bonati@df.unipi.it

massimo.delia@unipi.it

\$andrea.rucci@pi.infn.it

${ }^{1}$ For reference, a magnetic field of the order of $10^{15}$ Tesla corresponds to $e B \simeq 3.3 m_{\pi}^{2} \approx 0.06 \mathrm{GeV}^{2}$.
}

of the collision. Various studies have approached the issue of quarkonia spectra and production rates in the presence of magnetic backgrounds [11-17]. Many interesting phenomena have been predicted, including the emergence of magnetic field induced mixings between different states and of production anisotropies with respect to the collision plane.

The starting point for most of these investigations is the coupling of the magnetic field with electric charges and magnetic moments carried by the valence quarks. However, the magnetic field is known to induce important modifications also at a nonperturbative level and in the gluon sector: a natural question is whether that can change the picture both quantitatively and qualitatively.

A very interesting phenomenon in this respect is the magnetic field induced modification of the static quarkantiquark $(Q \bar{Q})$ potential. This is a typical pure gauge quantity (it is related to the expectation values of Wilson loops) which represents the starting point for many approaches to the study of heavy quarkonia, typically within a nonrelativistic approximation. The static potential is usually expressed in terms of the so-called Cornell parametrization [18]:

$$
V(r)=-\frac{\alpha}{r}+\sigma r
$$

where $\alpha$ is the Coulomb coupling and $\sigma$ the string tension.

Such a nonrelativistic approach is reliable only for heavy quark bound states, for which the interaction energy is not a large fraction of the total mass, and for not too large magnetic fields. As a rule of thumb one can use $e B \hbar /\left(m^{2} c^{3}\right) \ll 1$ (where $m$ is the heavy quark mass) to 
estimate which magnetic fields can be explored in this approach. In our worst case (charm quark and $|e| B=0.3 \mathrm{GeV}^{2}$ ) the previous ratio is about 0.18 , a numerical value which is roughly equal to the ratio $\left(M_{J / \Psi}-2 m_{c}\right) / M_{J / \Psi}$, which gives an estimate of the validity of the nonrelativistic approach also for $B=0$. For bottomonia states $e B \hbar /\left(m^{2} c^{3}\right)$ gets smaller by more than one order of magnitude.

The magnetic background field breaks rotational invariance, hence one may expect the emergence of anisotropies in the potential, which is central otherwise. The issue is clearly related to quark loop effects, since gluons are not directly coupled to the magnetic field, and has been investigated within various model studies [19-25]: the Coulomb coupling has been predicted to change in the transverse direction [19,22], in the longitudinal direction $[20,23]$ or in both [25]; regarding the string tension, while string theory studies do not predict an influence of the magnetic field on it [26-30], other approaches do (see, e.g., Refs. [21,25]).

Further insight into the question has been provided by a recent lattice QCD investigation [31]: the potential gets steeper in the directions transverse to the magnetic field and flatter in the longitudinal one. In particular one observes a larger (smaller) string tension in the transverse (longitudinal) direction, while the opposite happens for the Coulomb coupling [31].

The purpose of this study is to reconsider the computation of the heavy quarkonia spectrum and mixings in the presence of a magnetic field, in the light of the existing anisotropy in the static quark-antiquark potential. Since standard magnetic field effects decrease with the mass of the valence quarks, while the static potential remains unchanged, we might expect that corrections be relatively more important for bottomonia than for charmonia.

We will follow the standard nonrelativistic two-body approach (see, e.g., Refs. [32,33] for reviews on this subject), which can be tackled using standard numerical methods. In particular, our treatment will be close to that of Ref. [13], apart from the presence of the anisotropy, which will be included according to the lattice results of Ref. [31]. Since in Ref. [31] the potential has been studied only along two directions (transverse and longitudinal), we shall adopt the simplest possible parametrization which takes into account generic angles.

The paper is organized as follows. In Sec. II we will review the nonrelativistic approach to the two-body problem in an external magnetic field. In Sec. III we will discuss the form of the static potential in a magnetic background and our parametrization for its angular dependence. In Sec. IV we will discuss our numerical approach to the problem (more details and results from a test on the harmonic oscillator are reported in the Appendix) and present results for charmonia and bottomonia. Finally, in Sec. V, we will draw our conclusions.

\section{THE QUANTUM TWO-BODY PROBLEM IN EXTERNAL MAGNETIC FIELD}

The nonrelativistic Hamiltonian of two particles of masses $m_{i}$ and charges $q_{i}(i=1,2)$ in an external magnetic field is

$$
\hat{H}=\sum_{i=1}^{2} \frac{1}{2 m_{i}}\left[\hat{\boldsymbol{p}}_{i}-q_{i} \boldsymbol{A}\left(\boldsymbol{x}_{i}\right)\right]^{2}+V\left(\boldsymbol{x}_{1}, \boldsymbol{x}_{2}\right)-\left(\boldsymbol{\mu}_{1}+\boldsymbol{\mu}_{2}\right) \cdot \boldsymbol{B},
$$

where $\boldsymbol{\mu}_{1}, \boldsymbol{\mu}_{2}$ are the magnetic moments of the two particles. The presence of a vector potential $\boldsymbol{A}(\boldsymbol{x})$ makes the system not invariant under translations, i.e. $\boldsymbol{x}_{i} \rightarrow \boldsymbol{x}_{i}+\boldsymbol{\alpha}$. Even in the case of a uniform magnetic field, both the canonical and the kinetic momentum $\hat{\boldsymbol{P}}=\sum_{i=1}^{2}\left(\hat{\boldsymbol{p}}_{i}-q_{i} \boldsymbol{A}\left(\boldsymbol{x}_{i}\right)\right)$ do not commute with the Hamiltonian in Eq. (2).

An invariance group of $\hat{H}$ is obtained by simultaneously performing a coordinate translation and a gauge transformation, i.e. by changing both $\boldsymbol{x}_{i}$ and $\boldsymbol{p}_{i}$. The generator of this transformation is the pseudomomentum operator (see [34] for more details) and can be written in a particularly simple form in the symmetric gauge g $^{2}(\boldsymbol{x})=\frac{1}{2} \boldsymbol{B} \times \boldsymbol{x}$, where it is given by:

$$
\hat{\boldsymbol{K}}=\sum_{i=1}^{2}\left(\hat{\boldsymbol{p}}_{i}+\frac{1}{2} q_{i} \boldsymbol{B} \times \boldsymbol{x}_{i}\right)
$$

From this expression, it is not obvious that different components of the pseudomomentum are commuting observables, and in fact this is not in general true. This is however what happens for globally neutral system (in particular for the $Q \bar{Q}$ system to be studied in this paper), since the following commutation relations hold

$$
\left[\hat{K}_{j}, \hat{K}_{\ell}\right]=-i\left(q_{1}+q_{2}\right) \epsilon_{j \ell k} B_{k} .
$$

From now on we will explicitly restrict to the case of a particle-antiparticle system and we will adopt the notation $q \equiv q_{1}=-q_{2}$ for the electric charge and $m$ for the particle masses (see e.g. Ref. [13] for the general case). Written in terms of the relative coordinate $\boldsymbol{r}=\boldsymbol{x}_{1}-\boldsymbol{x}_{2}$, the pseudomomentum takes the form

$$
\hat{\boldsymbol{K}}=\hat{\boldsymbol{p}}_{1}+\hat{\boldsymbol{p}}_{2}+\frac{1}{2} q \boldsymbol{B} \times \boldsymbol{r}=\hat{\boldsymbol{P}}+q \boldsymbol{B} \times \boldsymbol{r},
$$

From this expression it appears natural to adopt the following ansatz for the eigenstate $\Phi$ of the two body Hamiltonian:

\footnotetext{
${ }^{2}$ See [35] for the form of the pseudomomentum in a generic gauge.
} 


$$
\Phi(\boldsymbol{R}, \boldsymbol{r}, \boldsymbol{\sigma})=\exp \left[i\left(\boldsymbol{K}-\frac{1}{2} q \boldsymbol{B} \times \boldsymbol{r}\right) \cdot \boldsymbol{R}\right] \Psi(\boldsymbol{r}, \boldsymbol{\sigma}),
$$

where $\boldsymbol{K}$ denotes the eigenvalue of the operator $\hat{\boldsymbol{K}}, \boldsymbol{R}$ is the coordinate of the center of mass and $\boldsymbol{\sigma}$ is a shorthand for the spin variables. Using the expression Eq. (6), the Hamiltonian in Eq. (2) can be rewritten in the form (acting on the reduced wave function $\Psi$ ):

$$
\begin{aligned}
\hat{H}= & \frac{\boldsymbol{K}^{2}}{2 M}-\frac{q}{M}(\boldsymbol{K} \times \boldsymbol{B}) \cdot \boldsymbol{r}-\frac{\nabla^{2}}{2 \mu} \\
& +\frac{q^{2}}{2 \mu}(\boldsymbol{B} \times \boldsymbol{r})^{2}+V(\boldsymbol{r})-\left(\boldsymbol{\mu}_{1}+\boldsymbol{\mu}_{2}\right) \cdot \boldsymbol{B},
\end{aligned}
$$

where $M \equiv 2 m$ and $\mu \equiv m / 2$ are, respectively, the total and the reduced mass of the two body system. Because of the lack of translation invariance, in this Hamiltonian the center of mass and the relative motion are not decoupled, unless $\boldsymbol{K} \times \boldsymbol{B}=0$.

Given this nontrivial dependence on the $\boldsymbol{K}$ value, one needs a prescription to subtract the energy associated with the center of mass motion. We will follow the idea presented in Ref. [13]: given the eigenvalue $E$ of Eq. (7), we will consider $E-\frac{\langle\hat{\boldsymbol{P}}\rangle^{2}}{2 M}$ as the corrected binding energy, with the kinetic momentum expectation value being computed by using Eq. (5).

Finally, let us discuss the role of the spin interaction term in Eq. (7). Quark magnetic moments may be expressed as $\boldsymbol{\mu}_{i}=g \mu_{i} \boldsymbol{s}_{i}$, where $g=2$ is the quark $g$-factor in the nonrelativistic approximation, $\mu_{i}=q_{i} / 2 m_{i}$ is the quark magneton and $s_{i}=\sigma^{i} / 2$ is its spin. Therefore, in the case of a quark-antiquark pair

$$
-\left(\boldsymbol{\mu}_{1}+\boldsymbol{\mu}_{2}\right) \cdot \boldsymbol{B}=-\frac{g q}{4 m}\left(\boldsymbol{\sigma}^{1}-\boldsymbol{\sigma}^{2}\right) \cdot \boldsymbol{B} .
$$

It can be verified that this term induces a mixing between the singlet $|00\rangle$ and the triplet $|10\rangle$ spin states. Indeed, in the presence of a magnetic field $\boldsymbol{B}=B \hat{z}$, the operator in Eq. (8) has the only nonzero matrix element between spin states given by

$$
\left\langle 00\left|\left(\boldsymbol{\mu}_{1}+\boldsymbol{\mu}_{2}\right) \cdot \boldsymbol{B}\right| 10\right\rangle=-\frac{g q B}{2 m} .
$$

Such a term increases the energy of the triplet state and decreases that of the singlet. When Eq. (9) is used for a noncentral potential, or when a spin-spin interaction is also present, some caution is needed, since possible mixings between different orbital states can be present (see Appendix for more details).

\section{THE $Q \bar{Q}$ POTENTIAL}

It is well known that the main features of the quarkonium spectrum can be understood by using a central potential between the heavy quarks of the so-called Cornell form reported in Eq. (1). This parametrization was introduced in Ref. [18] on phenomenological bases, as the simplest form of the potential that takes into account both the short distance perturbative contribution and color confinement. It was later realized that the form Eq. (1) of the potential correctly describes the spin averaged potential between two static (i.e. infinitely massive) quarks as estimated from the first principles of QCD by its lattice formulation (see, e.g., Ref. [32]).

To properly describe the fine structure of the quarkonium spectrum further spin dependent terms have to be added to the potential. All such corrections to the static quark potential can in principle be obtained by an expansion in the inverse quark mass (see Ref. [33]), however their precise functional form is generally not well known and difficult to extract from LQCD computations. Because of this lack of precise theoretical information, several different parametrizations of these terms exist, whose coefficients are fixed by comparing with experimental results.

Since we are mainly interested in the lowest quarkonium levels (see Table I), the most important contribution to be added to the Cornell potential is the spin-spin interaction, responsible, e.g., for the mass splitting between the $J / \Psi$ and $\eta_{c}$ levels of charmonium. We will adopt the parametrization

$$
V_{\sigma \sigma}=\left(\boldsymbol{\sigma}_{1} \cdot \boldsymbol{\sigma}_{2}\right) \gamma e^{-\beta r},
$$

where $\beta$ and $\gamma$ are phenomenological constants, whose values are reported in Table III. This form of the spin-spin

TABLE I. Lowest part of charmonium and bottomonium mass spectrum. Data from [37].

\begin{tabular}{lccc}
\hline \hline$Q \bar{Q}$ & State & Name & Mass [MeV] \\
\hline$c \bar{c}$ & $1^{1} S_{0}$ & $\eta_{c}$ & $2980.3 \pm 1.2$ \\
$\cdot \cdot$ & $1^{3} S_{1}$ & $J / \psi$ & $3096.916 \pm 0.011$ \\
$\cdot$ & $1^{3} P_{0}$ & $\chi_{c 0}$ & $3414.75 \pm 0.31$ \\
$\cdot$ & $1^{3} P_{1}$ & $\chi_{c 1}$ & $3510.66 \pm 0.07$ \\
. & $1^{3} P_{2}$ & $\chi_{c 2}$ & $3556.20 \pm 0.09$ \\
. & $1^{1} P_{1}$ & $h_{c}$ & $3525.38 \pm 0.11$ \\
$\cdot$ & $2^{1} S_{0}$ & $\eta_{c}(2 S)$ & $3639.4 \pm 1.3$ \\
. & $2^{3} S_{1}$ & $\psi(2 S)$ & $3686.109 \pm 0.02$ \\
$b \bar{b}$ & $1^{1} S_{0}$ & $\eta_{b}$ & $9390.9 \pm 2.8$ \\
$\cdot$ & $1^{3} S_{1}$ & $\Upsilon$ & $9460.30 \pm 0.26$ \\
$\cdot$ & $1^{3} P_{0}$ & $\chi_{b 0}$ & $9859.44 \pm 0.74$ \\
$\cdot$ & $1^{3} P_{1}$ & $\chi_{b 1}$ & $9892.78 \pm 0.57$ \\
$\cdot$ & $1^{3} P_{2}$ & $\chi_{b 2}$ & $9912.21 \pm 0.57$ \\
. & $1^{1} P_{1}$ & $h_{b}$ & $9898.3 \pm 1.1$ \\
. & $2^{1} S_{0}$ & $\eta_{b}(2 S)$ & $9999.0 \pm 3.5$ \\
. & $2^{3} S_{1}$ & $\Upsilon(2 S)$ & $10232.26 \pm 0.31$ \\
\hline \hline
\end{tabular}


interaction is supported by the lattice result presented in Ref. [36] and was previously used in Ref. [13] to study the influence of a magnetic field on the meson spectrum.

While in previous studies the static Cornell potential in Eq. (1) was assumed to be independent of the magnetic field, in this work we will consider also the anisotropy observed in Ref. [31]. However, since in Ref. [31] only the values of the potential along the coordinate axes have been investigated, we are forced to make an ansatz on the form of the potential, with the constraint that it reproduces the observed behavior on the axes. To this aim, we propose the following anisotropic form of the static potential terms ( $\boldsymbol{B}$ is directed along the $\hat{z}$ axis)

$$
\begin{gathered}
\frac{\alpha}{r} \rightarrow \frac{\alpha}{\sqrt{\epsilon_{x y}^{(\alpha)}\left(x^{2}+y^{2}\right)+\epsilon_{z}^{(\alpha)} z^{2}}} \\
\sigma r \rightarrow \sigma \sqrt{\epsilon_{x y}^{(\sigma)}\left(x^{2}+y^{2}\right)+\epsilon_{z}^{(\sigma)} z^{2}}
\end{gathered}
$$

where the scaling parameters $\epsilon_{x y}(B)$ and $\epsilon_{z}(B)$ are functions of the external magnetic field. Such a parametrization is inspired by that of the electrostatic interaction in the presence of an anisotropic dielectric constant (see Ref. [38], Sec. 13). It is possible to rewrite the expression above in a more usual fashion, by absorbing the angular and $B$ dependences into the $\alpha$ and $\sigma$ parameters

$$
V(r, \theta, B)=-\frac{\alpha(\theta, B)}{r}+\sigma(\theta, B) r
$$

where $\theta$ is the spherical azimuthal angle and

$$
\begin{aligned}
& \alpha(\theta, B)=\frac{\alpha}{\epsilon_{1}^{(\alpha)} \sqrt{1+\epsilon_{2}^{(\alpha)}(B) \sin ^{2}(\theta)}} \\
& \sigma(\theta, B)=\sigma \epsilon_{1}^{(\sigma)}(B) \sqrt{1+\epsilon_{2}^{(\sigma)}(B) \sin ^{2}(\theta)}
\end{aligned}
$$

with $\epsilon_{i}^{(O)}$ related to the scaling parameters previously defined by setting $\epsilon_{1}=\sqrt{\epsilon_{z}}$ and $\epsilon_{2}=\epsilon_{x y} / \epsilon_{z}-1$. According to Ref. [31], when increasing the magnetic field the string tension gets larger in the transverse directions, while it decreases in the longitudinal $\hat{z}$ direction; the opposite behavior is observed for the coefficient $\alpha$ of the Coulomb term. To reproduce such a behavior, the coefficients $\epsilon_{i}^{(O)}$ must be positive. In particular, they are related to the coefficients $A, C$ estimated in Ref. [31] (see Table II) by the relations

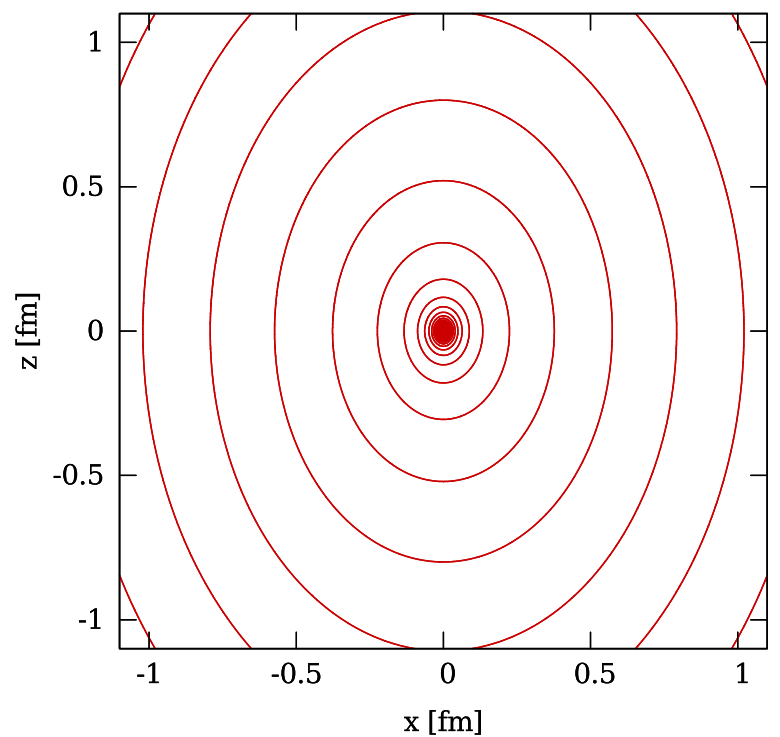

FIG. 1 (color online). Contour map of the potential in Eq. (12) on the $x-z$ plane for $B=0.6 \mathrm{GeV}^{2}$, with $\boldsymbol{B}$ directed along the $\hat{z}$ axis. Parameters used are the ones for charmonium (see Table III).

$$
\begin{aligned}
\epsilon_{1}^{(\alpha)}(B) & =\left(1+A^{\alpha_{z}}(|e| B)^{C^{\alpha_{z}}}\right)^{-1} \\
\epsilon_{2}^{(\alpha)}(B) & =\left(\frac{1+A^{\alpha_{z}}(|e| B)^{C^{\alpha_{z}}}}{1+A^{\alpha_{x y}}(|e| B)^{C^{\alpha y}}}\right)^{2}-1 \\
\epsilon_{1}^{(\sigma)}(B) & =\left(1+A^{\sigma_{z}}(|e| B)^{C^{\sigma_{z}}}\right) \\
\epsilon_{2}^{(\sigma)}(B) & =\left(\frac{1+A^{\sigma_{x y}}(|e| B)^{C_{x y}}}{1+A^{\sigma_{z}}(|e| B)^{C^{\sigma_{z}}}}\right)^{2}-1 .
\end{aligned}
$$

A graphical representation of the anisotropic potential is shown in Fig. 1.

Finally, let us briefly discuss the role of the spin-spin interaction term (10) within the anisotropic potential picture. In principle, both parameters, $\beta$ and $\gamma$, may acquire a dependence on the external magnetic field like that observed for the string tension and the Coulomb coupling. Such a dependence has not yet been investigated and no ansatz is available. However, since this term represents a spin-dependent relativistic correction of order $\mathrm{m}^{-2}$ $[32,33,39,40]$, it is reasonable to expect that also the $B$ dependence be of the same order in the quark mass $m$, hence much weaker than that induced on the spinindependent part of the potential. Therefore in the following we will make use of the same values for $\beta$ and $\gamma$ adopted in Ref. [13]. A similar attitude will be adopted with respect to other relativistic corrections, like the spin-orbit term.

\section{COMPUTATION AND NUMERICAL RESULTS}

In this section we report our results for the dependence on the magnetic field of the masses of the $1 S$ and $1 P$ states for both charmonium and bottomonium. 
TABLE II. Coefficients $A, C$ estimated in [31].

\begin{tabular}{lrccc}
\hline \hline$O$ & \multicolumn{1}{c}{$A^{O_{x y}}$} & \multicolumn{1}{c}{$C^{O_{x y}}$} & $A^{O_{z}}$ & \multicolumn{1}{c}{$C^{O_{z}}$} \\
\hline$\sigma$ & $0.29 \pm 0.02$ & $0.9 \pm 0.1$ & $-0.34 \pm 0.01$ & $1.5 \pm 0.1$ \\
$\alpha$ & $-0.24 \pm 0.04$ & $0.7 \pm 0.2$ & $0.24 \pm 0.03$ & $1.7 \pm 0.4$ \\
\hline \hline
\end{tabular}

To this purpose we used the Hamiltonian in Eq. (7), with a magnetic field $\boldsymbol{B}=B \hat{\boldsymbol{z}}$ and pseudomomentum $\boldsymbol{K}=K \hat{\boldsymbol{x}}$, with the anisotropic potential parametrized as in Eq. (12), together with the spin-spin interaction term Eq. (10). Values of the parameters adopted are reported in Table III and have been fixed according to Ref. [13], thus enabling a direct comparison with the case in which the anisotropy of the potential is neglected. Numerical values of the anisotropy parameters, defined in Eq. (14), have been fixed by using the coefficients shown in Table II. To take into account the uncertainty associated with these parameters, several simulations were performed, corresponding to different combinations of these values.

In order to allow for a better physical comprehension of the results, the mass spectrum will be shown as a function of the mean kinetic momentum $\langle\boldsymbol{P}\rangle$, instead of using the pseudomomentum $\boldsymbol{K}$. To this purpose we solved the system for several values of $\boldsymbol{K}$, computed the value of the mean kinetic momentum for each eigenstate and interpolated these values to the desired point. Details of the numerical algorithm used to extract eigenstates are reported in the Appendix, together with a test of the algorithm in an analytically solvable case.

\section{A. Charmonium states}

We start by studying the $\eta_{c}$ and $J / \psi$ states, which correspond to the $1 S$ states of the charmonium at vanishing magnetic field. The experimental masses of these states are well reproduced by using the potential parameter reported in Table III and their behavior as a function of the external magnetic field $B$ is shown in Fig. 2. The spin components $s_{z}= \pm 1$ of the $J / \psi$ stay degenerate also for $B \neq 0$ but they split from the $s_{z}=0$ component, which mixes with the $\eta_{c}$ state.

In order to isolate the contribution of the anisotropy in the potential, we reported in Fig. 2 also the masses computed without taking into account the anisotropy (i.e. with $\epsilon_{1}^{(\alpha)}=\epsilon_{1}^{(\sigma)}=1$ and $\epsilon_{2}^{(\alpha)}=\epsilon_{2}^{(\sigma)}=0$ ). It can be seen that in all cases the masses are increased by taking into account the anisotropy; as a consequence, for the $\eta_{c}$ the

TABLE III. Parameters used for the potential (same as in Ref. [13]).

\begin{tabular}{cccccc}
\hline \hline & $\gamma$ & $\beta$ & $\alpha$ & $\sigma$ & $m$ \\
\hline$c \bar{c}$ & $2.060 \mathrm{GeV}$ & $1.982 \mathrm{GeV}$ & 0.312 & $0.174 \mathrm{GeV}^{2}$ & $1.29 \mathrm{GeV}$ \\
$b \bar{b}$ & $0.318 \mathrm{GeV}$ & $1.982 \mathrm{GeV}$ & 0.421 & $0.210 \mathrm{GeV}^{2}$ & $4.70 \mathrm{GeV}$ \\
\hline \hline
\end{tabular}

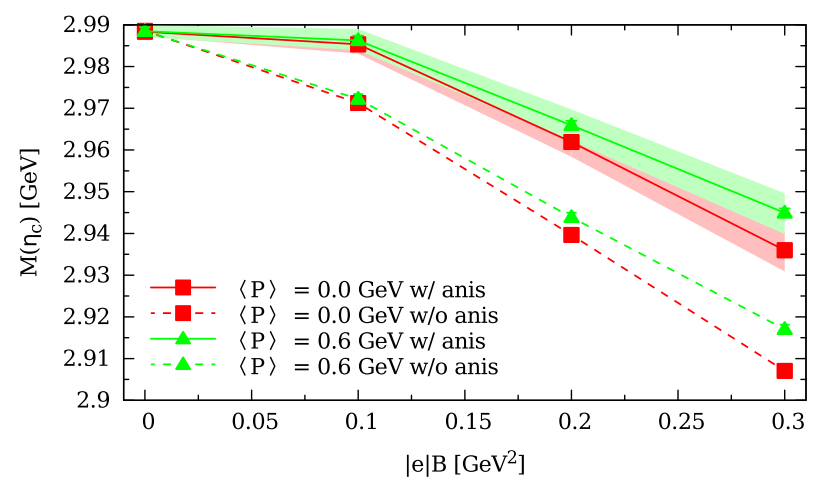

(a)

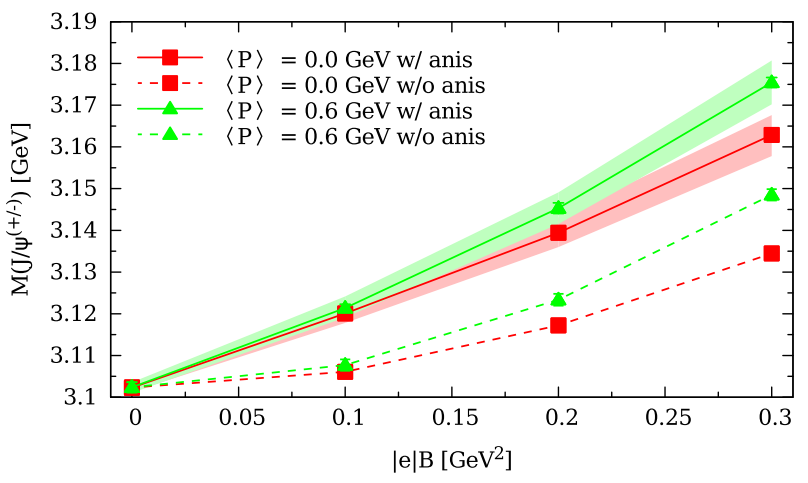

(b)

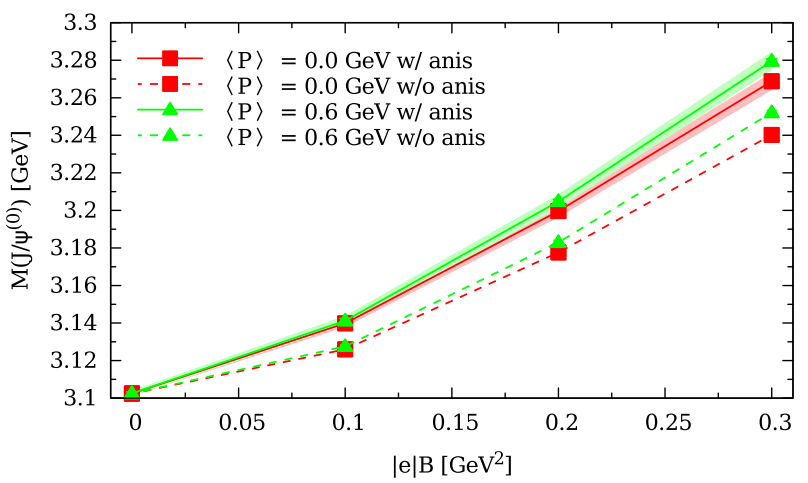

(c)

FIG. 2 (color online). Behavior of the masses of the charmonium $1 S$ states with respect to the magnetic field, both with and without the magnetic anisotropy in the potential and for two different values of $\langle P\rangle$. Data points represent the mass extracted making use of the central values of the parameters in Table II, while shaded regions take into account uncertainties on the parameters. (a) The $\eta_{c}$ singlet state. (b) The $s_{z}= \pm 1$ components of the $J / \psi$ state. (c) The $s_{z}=0$ component of the $J / \psi$ state.

dependence of the mass on $B$ gets milder, while the opposite effect is observed for the $J / \psi$ states. We note that for the $\eta_{c}$ and the $s_{z}= \pm 1$ components of the $J / \psi$ the effect induced by the anisotropy is of the same order of magnitude as the effect expected when no anisotropy is present. 


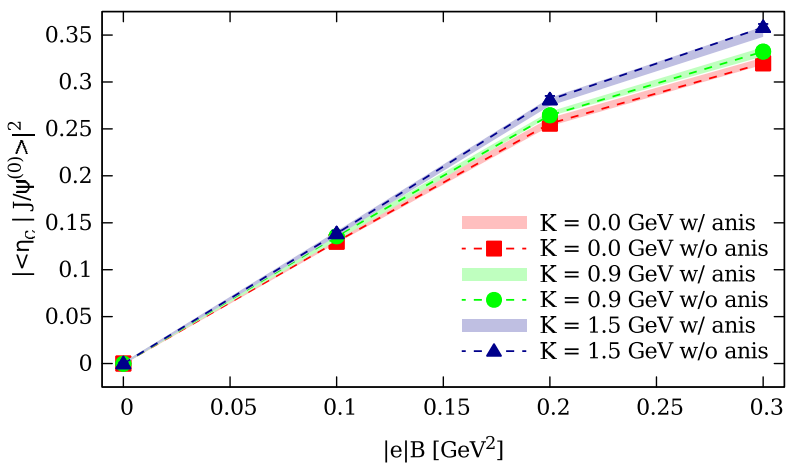

FIG. 3 (color online). Square modulus of the mixing between the $s_{z}=0$ component of the $J / \psi$ state and the $\eta_{c}$ state, both with and without the magnetic anisotropy in the potential and for several values of the pseudomomentum $\boldsymbol{K}$.

A nonvanishing kinetic momentum has an effect analogous to that of the anisotropy: it reduces the dependence on $B$ of the $\eta_{c}$ mass while it increases that of the $J / \psi$ states. This effect is however quite small for the values of the kinetic momenta explored and it is almost of the same magnitude of the uncertainties associated with the anisotropies. It cannot be excluded that this effect increases for larger momenta, but to systematically explore this regime a fully relativistic treatment would be required.

As previously noted, the magnetic field introduces a mixing between the $\eta_{c}$ state and the $s_{z}=0$ component of the $J / \psi$ state. The behavior of this mixing with respect to the magnetic field and the pseudomomentum $K$ is shown in Fig. 3 while the dependence on the magnetic field is quite strong, the mixing turn out to be almost independent of the value of the pseudomomentum. Moreover, contrary to what happens for the masses, the mixing is almost insensitive to the anisotropy of the potential.

We now present some preliminary data for the $1 P$ states. In this case the fine spectrum of known $1 P$ charmonium

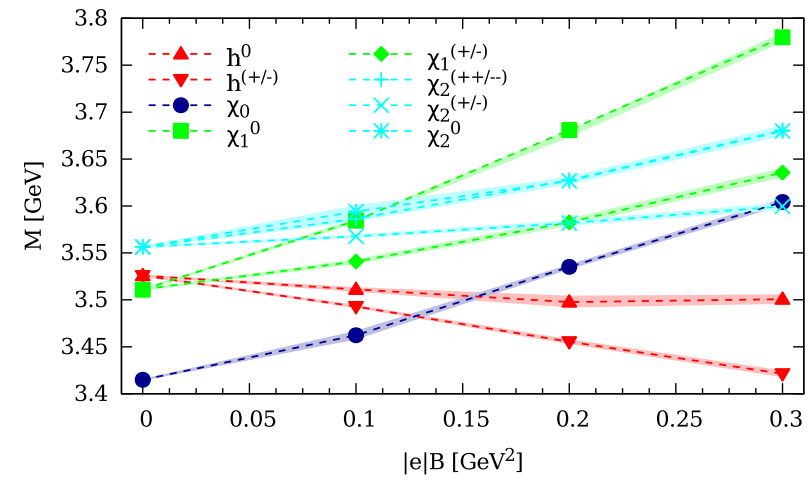

FIG. 4 (color online). Behavior of the masses of the charmonium $1 P$ excited states with respect to the magnetic field. We report for simplicity only data obtained by using the anisotropic form of the potential, the general features of this picture are present also in the standard case of the central potential. levels is not correctly reproduced for $B=0$, a fact that is likely due to the absence of the spin-orbit coupling and other relativistic corrections in the used Hamiltonian. If we assume that these relativistic terms do not depend on the external magnetic field, the computed variation of the mass $\Delta m(B)$ due to a nonvanishing $B$ can be used to shift the known $B=0$ values of the masses to obtain the spectrum at $B \neq 0$. It is clear that this is an approximate procedure and that a more precise study will be required to quantify the systematic error introduced in this way; nevertheless this

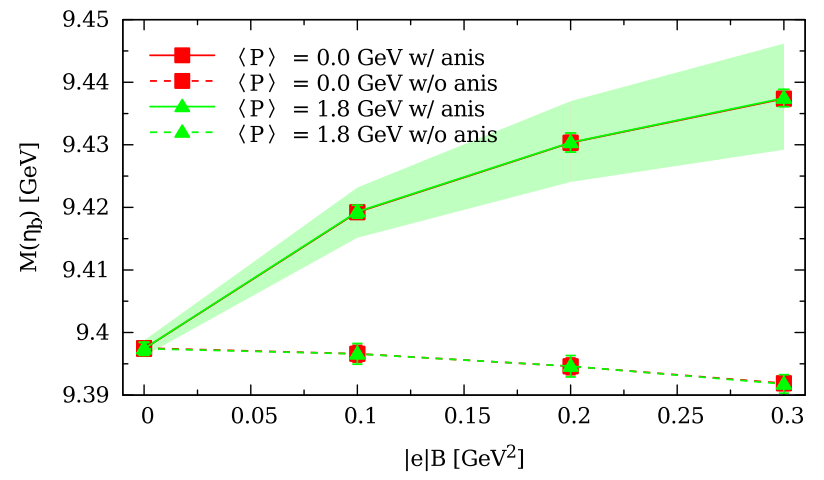

(a)

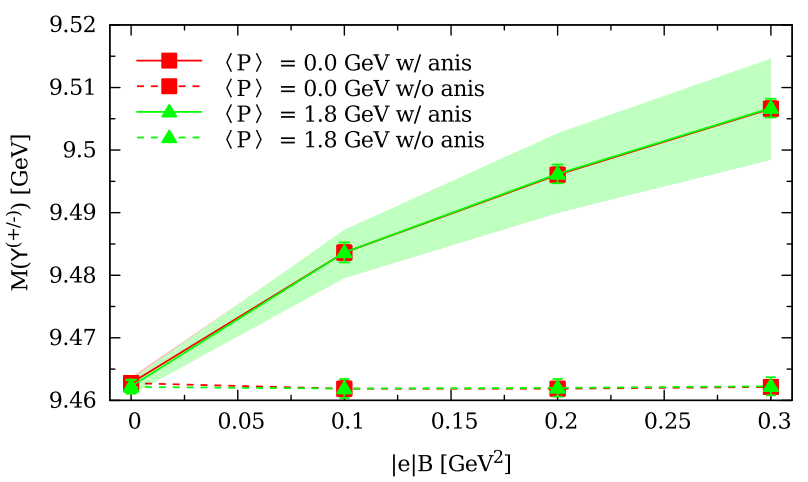

(b)

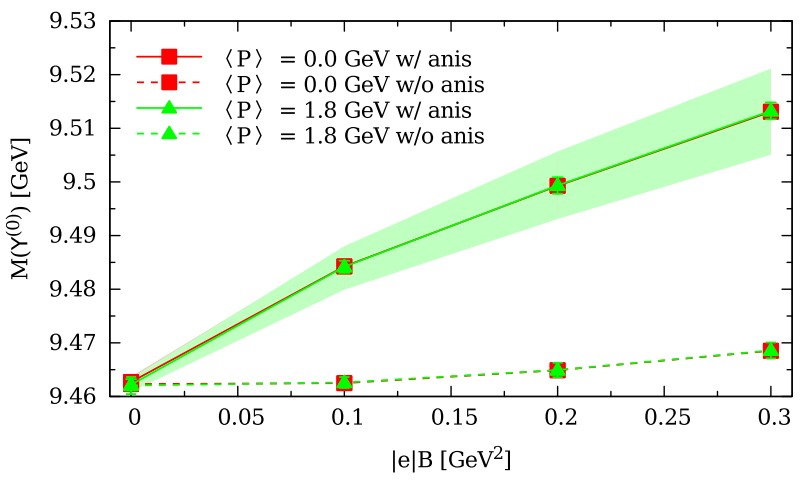

(c)

FIG. 5 (color online). Behavior of the masses of the bottomonium $1 S$ states with respect to the magnetic field, both with and without the magnetic anisotropy in the potential and for several values of $\langle P\rangle$. (a) The $\eta_{b}$ singlet state. (b) The $s_{z}= \pm 1$ components of the $\Upsilon$ state. (c) The $s_{z}=0$ component $\Upsilon$ state. 
HEAVY QUARKONIA IN STRONG MAGNETIC FIELDS

method is expected to give reliable information in the limit of large quark mass $m$, since relativistic effects are suppressed by inverse powers of $m$. The results obtained in this way are shown in Fig. 4 and the main difference with respect to the $1 S$ case is that in the $1 P$ case several level crossings happen by increasing the magnetic field. From Figs. 4 and 2 it can be seen that the gap between the $h$ states and the $J / \psi$ states is strongly reduced by increasing the magnetic field, a fact that may have significant consequences on branching ratios of the $h$ meson.

\section{B. Bottomonium states}

The effect of the anisotropy in the potential on bottomonium states is qualitatively similar to that on charmonium states, however its quantitative relevance is much larger, as can be seen from the spectrum of the $1 S$ states in Fig. 5 the mass shift due to the anisotropy is about one order of magnitude larger than the one due to quark magnetic momenta only. This is not unexpected, since the effect of the anisotropy in the potential is independent of the quark masses, while quark magnetic momenta go to zero like $1 / \mathrm{m}$. As a consequence the relative effect of the anisotropy is much stronger for bottomonium than for charmonium.

In Fig. 6 we show the behavior of the mixing between the singlet state $\eta_{b}$ and the $s_{z}=0$ component of the $\Upsilon$ as a function of the magnetic field. Although the value of the mixing is reduced by about a factor $3\left(\approx m_{b} / m_{c}\right)$ with respect to the charmonium case, its value is still largely independent on the anisotropy.

A preliminary analysis of the $1 P$ states is shown in Fig. 7 it is obtained by the same procedure adopted for the charmonium, which for bottomonium is expected to be more reliable. Also for bottomonium some level crossings can be seen, however in this case the uncertainties, due to the propagation of the error on the anisotropy, become quite large with respect to the typical level separations.

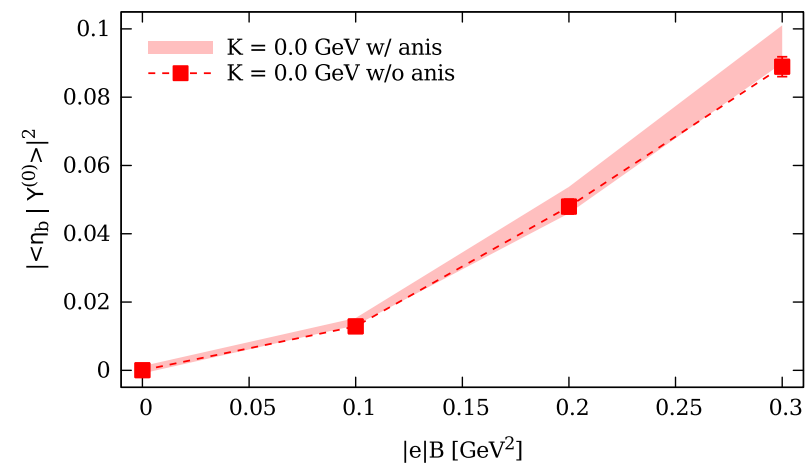

FIG. 6 (color online). Square modulus of the mixing between the $s_{z}=0$ component of the $\Upsilon$ state and the $\eta_{b}$ state, both with and without the magnetic anisotropy in the potential and for several values of the pseudomomentum $\boldsymbol{K}$.
PHYSICAL REVIEW D 92, 054014 (2015)

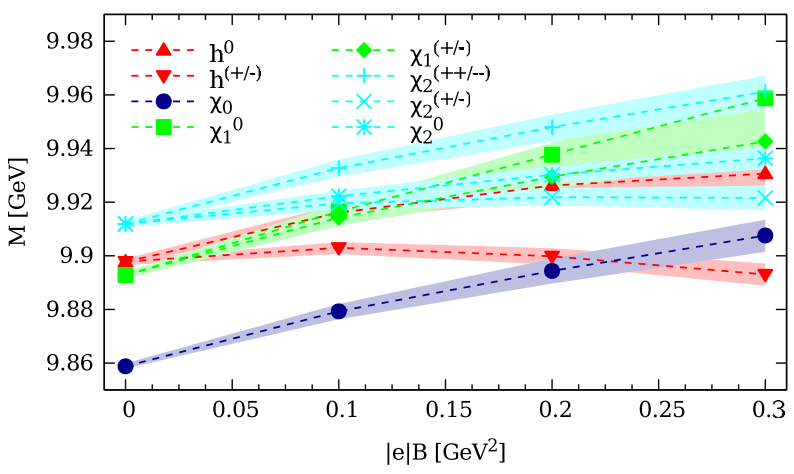

FIG. 7 (color online). Behavior of the masses of the bottomonium $1 P$ excited states with respect to the magnetic field.

\section{SUMMARY AND CONCLUSIONS}

The main purpose of this work has been the study of the possible influence of strong external magnetic fields on the heavy-meson mass spectrum. Following the approach of Ref. [13], we used a nonrelativistic model to extract the lowest lying $c \bar{c}$ and $b \bar{b}$ states and the magnetic field induced mixings among them. As a new ingredient, we considered the anisotropies induced by the magnetic field at a nonperturbative level in the static quark-antiquark potential, as determined by a recent LQCD investigation [31]. Moreover, we extended our analysis to the first excited $1 P$ states.

Various approximations are involved in our computation: relativistic corrections were only partially included and any possible dependence of their magnitude on $B$ was neglected. Moreover, the exact angular dependence of the static potential was obtained by a suitable interpolation of data reported in Ref. [31].

A first issue of phenomenological interest regards the mixing between different states, since that should lead to a modification of both decay patterns and production rates. For instance, results suggest a contamination of the semileptonic decay channels between the $\eta_{c}$ and the $J / \psi$ and between the $\eta_{b}$ and $\Upsilon$ mesons. However, in this case such mixings are observed even without taking into account the potential anisotropy [13], and we have verified that its inclusion does not lead to significant quantitative changes.

On the other hand, results suggest that the presence of the anisotropy in the potential has significant effects on the heavy-meson mass spectrum. An increase of the masses is generally observed, with respect to the case in which the anisotropy is not included, the increase being of the order of $30-40 \mathrm{MeV}$ at the maximum magnetic field explored, $|e| B \simeq 0.3 \mathrm{GeV}^{2}$. The effect is more dramatic for bottomonium states, where it can even change, in some cases, the sign of the dependence of the mass on $B$ : the reason is that standard magnetic interaction terms in the Hamiltonian of the system [see Eq. (7)] are suppressed by the inverse of the quark mass, while the nonperturbative effect on the 
static potential is mass independent, hence it becomes dominant for bottomonium.

Finally, a new effect pointed out by our study regards the possible crossings of $1 P$ states as a function of $B$. Such crossings are observed even without taking into account the anisotropy of the potential, however its presence makes them clearer. We stress that our results for $1 P$ states are still preliminary: in particular, regarding the level crossings, we assumed the experimentally observed spectrum at $B=0$, which can be reproduced for such states only by taking into account relativistic corrections like the spin-orbit one. On the other hand, the spin-orbit term was not taken into account to compute the $B$-dependence of the spectrum, therefore a significant $B$-induced correction to this term could change the scenario.

One should consider that present results are obtained using the $T=0$ form of the potential and assuming a constant and uniform magnetic field. The use of the $T=0$ potential is justified only for hard processes taking place in the initial stages of noncentral collisions, before the strongly interacting medium thermalizes. Future lattice simulations could provide information on magnetic field effects at finite $T$, which could be relevant to production and decay rates of heavy quark states in the thermalized medium; instead, it will be relatively more difficult to take into account the possible effects of inhomogeneities or time dependence of the magnetic field distribution. Further improvement on present results could also be obtained once lattice simulations provide information about the exact angular dependence of the static potential (which in the present study was partially based on an ansatz) and, possibly, about the spin-dependent part of the potential.

\section{ACKNOWLEDGMENTS}

It is a pleasure to thank Michele Mesiti, Marco Mariti, Francesco Negro, Francesco Sanfilippo and Michael Strickland for useful comments and discussions. Numerical simulations have been performed using resources provided by the Scientific Computing Center at INFN-Pisa.

\section{APPENDIX: THE NUMERICAL ALGORITHM}

The algorithm used to numerically compute eigenvalues and eigenfunctions of the reduced Hamiltonian (7) is the finite difference time domain method (FDTD) described, e.g., in $[41,42]$. The main idea of this approach is the following: once the Schrödinger equation is Wick rotated to imaginary time

$$
\left(\frac{\partial}{\partial \tau}+\hat{H}\right) \Psi(\boldsymbol{r}, \tau)=0,
$$

the formal solution of the problem with initial condition $\Psi(\boldsymbol{r}, 0)=\zeta(\boldsymbol{r})$ is

$$
\Psi(\boldsymbol{r}, \tau)=\sum_{\alpha}\left\langle\Phi_{\alpha} \mid \zeta\right\rangle \Phi_{\alpha}(\boldsymbol{r}) e^{-\epsilon_{\alpha} \tau},
$$

where $\epsilon_{\alpha}$ and $\Phi_{\alpha}$ are the eigenvalues and eigenfunctions of the Hamiltonian. By looking at the large time behavior of $\Psi(\boldsymbol{r}, \tau)$ we can thus identify the lowest $\epsilon_{\alpha}$ (and the corresponding $\Phi_{\alpha}$ ) among those corresponding to states such that $\left\langle\Phi_{\alpha} \mid \zeta\right\rangle \neq 0$. By using an initial wave function $\zeta(\boldsymbol{r})$ with specific symmetries we can thus select the state we are interested in and, in this work, hydrogen-like wave functions were used to this purpose when $B=0$. States for $B \neq 0$ were extracted by adiabatically switching on the magnetic field in an initial stage of the evolution. This procedure turned out to work well for the low lying states, however numerical instabilities emerge when applying this technique to higher excited states.

From the numerical point of view the evolution is performed by introducing a temporal and a spatial lattice spacing (denoted by $d \tau$ and $a$, respectively) and by approximating derivatives in Eq. (A1) with finite differences. In this way variables at time $\tau+d \tau$ can be written in term of the ones at time $\tau$ (see [41] for details).

After every time evolution step we get an estimate of the bound state energy and of other properties of the state we want to study; the process ends when the variation of these quantities during a time interval $\tau_{s}$ goes below a fixed threshold. The time interval $\tau_{s}$ was set to $1 / M$, where $M$ is a rough estimate of the mass for the state we are interested in. The precision of $1 \mathrm{MeV}$ was used as a stopping criterion for the energy determination (this value can be used as the uncertainty in the energy computation).

The numerical algorithm just described concerns the orbital part of the Hamiltonian and can be applied, once the total spin is fixed, to the reduced Hamiltonian Eq. (7) (without the $\boldsymbol{\mu} \cdot \boldsymbol{B}$ terms) together with the $V_{\sigma \sigma}$ potential defined by Eq. (10). By using these eigenstates we can then evaluate the matrix elements of the operator $\left(\boldsymbol{\mu}_{1}+\boldsymbol{\mu}_{2}\right) \cdot \boldsymbol{B}$ and diagonalize this matrix to extract the final eigenvalues and eigenstates.

The operator $\left(\boldsymbol{\mu}_{1}+\boldsymbol{\mu}_{2}\right) \cdot \boldsymbol{B}$ has nonvanishing matrix elements only between the spin states $|00\rangle$ and $|10\rangle$ (see Ref. [13] for details), however some care is needed here since, due to the specific form of $V_{\sigma \sigma}$ adopted, nothing prevents a mixing between states with different orbital momenta to occur (this problem is relevant also for $B=0$ ). The effect of this mixing between different orbital momenta, however, turns out to be rather small: in all the studied cases the overlap $\langle\ell=0 \mid \ell=1\rangle$ was compatible with zero within machine precision and the overlap $\langle\ell=0 \mid \ell=2\rangle$ was at most of the percent level.

All the results presented in the main text were obtained by using a lattice of physical spatial extent $V=\left(30 \mathrm{GeV}^{-1}\right)^{3} \simeq(6 \mathrm{fm})^{3}$ and several values of the lattice spacings, in order to extract the continuum limit. The spatial lattice spacings used were 
HEAVY QUARKONIA IN STRONG MAGNETIC FIELDS

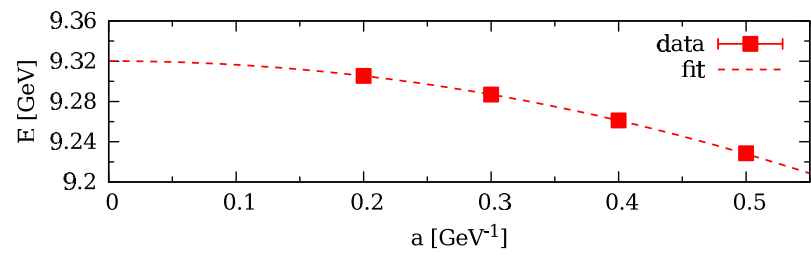

(a)

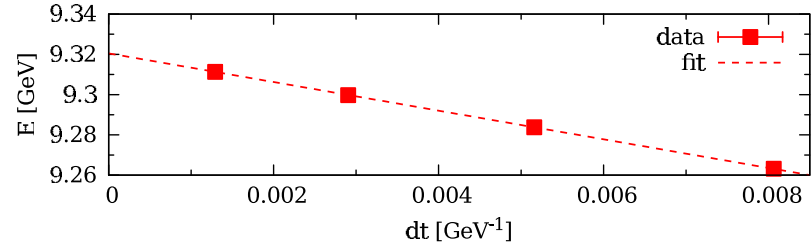

(b)

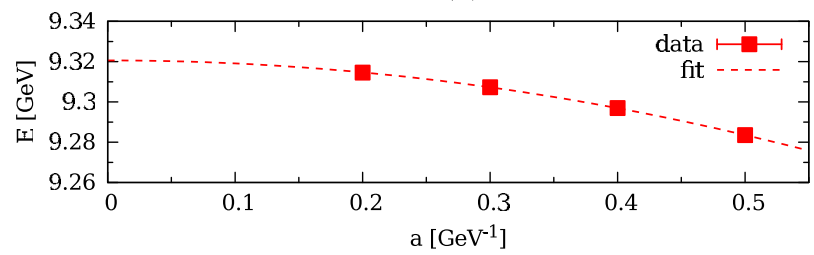

(c)

FIG. 8 (color online). Test of the continuum limit for parameters $\omega=1.5 \mathrm{GeV},|e| B=0.9 \mathrm{GeV}^{2}$ and $K=5.0 \mathrm{GeV}$. Data correspond to the energy of the eigenstate $\Phi_{(0,1)}^{( \pm)}$, while dashed lines are fit curves. (a) First $\mathrm{d} \tau \rightarrow 0$ and then $a \rightarrow 0$, the final continuum value is $E_{a}=9.3205(13) \mathrm{GeV}$. (b) First $a \rightarrow 0$ and then $\mathrm{d} \tau \rightarrow 0, E_{b}=9.3206(13) \mathrm{GeV}$. (c) $a \rightarrow 0$ with $a \propto d \tau^{2}$, $E_{c}=9.3201(12) \mathrm{GeV}$.

$a=0.250,0.375,0.500,0.625 \mathrm{GeV}^{-1}$ and the temporal lattice spacing was fixed by the relation $\mathrm{d} \tau=m a^{2} / 20$, $m$ being the quark mass.

\section{Test of the algorithm}

We report here the results of some tests performed to verify the correctness of the algorithm implementation. As a test bed, we used the case of the harmonic potential

$$
V(r)=\frac{1}{2} m \omega r^{2},
$$

which is analytically solvable (see Ref. [13,35]) and thus allows for a direct check of the numerical data. Following the notation of Ref. [13], eigenstates are classified by the quantum numbers $\boldsymbol{K}, n_{\perp}, n_{z}, \ell, s$ and $s_{z}$, where $\boldsymbol{K}$ is the pseudomomentum defined in Eq. (5), $s$ is the total spin of the system, $s_{z}$ its projection on the $z$-axis and $n_{\perp}, n_{z}, \ell$ are quantum numbers specific for the harmonic potential. For fixed spin $s$, the ground state will be denoted by $\Phi_{(0, s)}$ and
PHYSICAL REVIEW D 92, 054014 (2015)

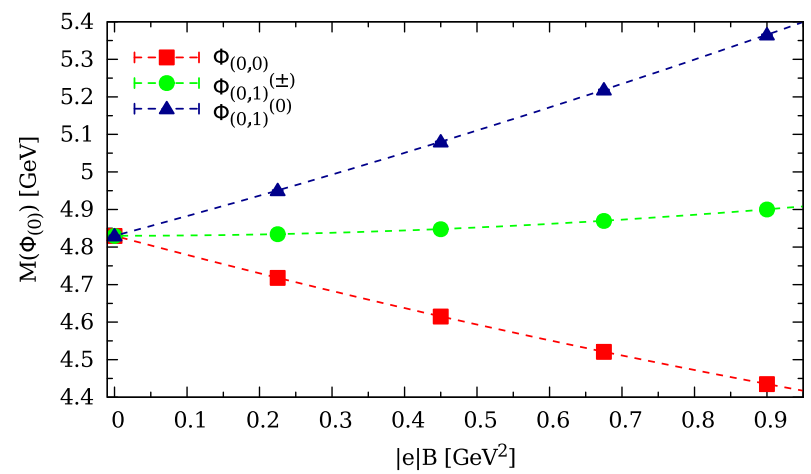

(a)

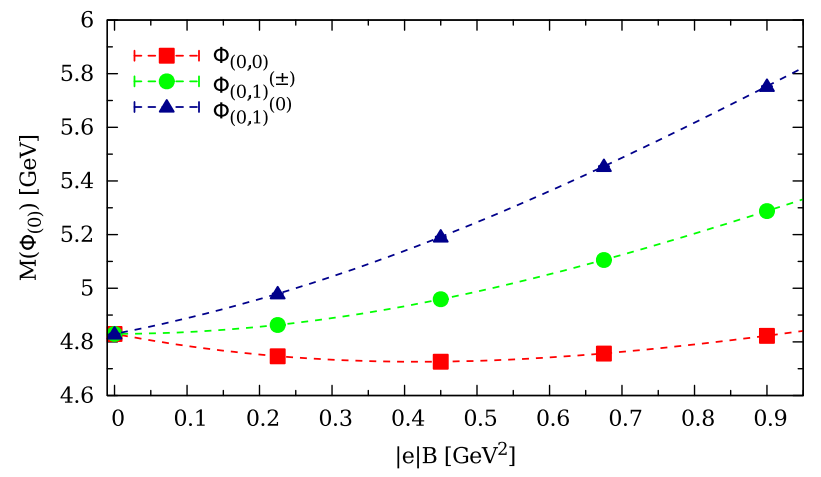

(b)

FIG. 9 (color online). Behavior of the masses for the eigenstates $\Phi_{(0, s)}$ with respect to the magnetic field. Dots correspond to numerical data extrapolated to the continuum, dashed curves represent the analytic expected behavior. (a) Data corresponding to $|\boldsymbol{K}|=0$. (b) Data corresponding to $|\boldsymbol{K}|=5 \mathrm{GeV}, \boldsymbol{K}=|\boldsymbol{K}| \hat{\boldsymbol{x}}$.

corresponds to the case $n_{\perp}=n_{z}=\ell=0$ (the superscripts \pm and 0 will be used to denote the $s_{z}$ components).

As a first test we verified that, for fixed physical parameters, the same result is obtained by performing the continuum limit in different ways: it is possible to first extract the limit for $a \rightarrow 0$ at fixed $\mathrm{d} \tau$ and then send $\mathrm{d} \tau$ to zerol; alternatively, it is possible to exchange the order of the limits or to perform both limits together. Since in our implementation discretization errors are linear in $\mathrm{d} \tau$ and quadratic in $a$, it is convenient to perform the limit by using a relation of the form $\mathrm{d} \tau \propto a^{2}$ with a fixed proportionality factor.

The three possible ways to continuum extrapolate are compared in Fig. 8 and they nicely agree with each other within errors. In Fig. 9 the final results (i.e. continuum extrapolated and taking into account spin mixing) for the energies of the low lying states are compared with the theoretical expectations, and in all cases a perfect agreement is found. 
[1] D. Kharzeev, K. Landsteiner, A. Schmitt, and H.-U. Yee, Strongly interacting matter in magnetic fields, Lect. Notes Phys. 871, 1 (2013).

[2] V. A. Miransky and I. A. Shovkovy, Quantum field theory in a magnetic field: From quantum chromodynamics to graphene and Dirac semimetals, Phys. Rep. 576, 1 (2015).

[3] R. C. Duncan and C. Thompson, Formation of very strongly magnetized neutron stars-Implications for gamma-ray bursts, Astrophys. J. 392, L9 (1992).

[4] T. Vachaspati, Magnetic fields from cosmological phase transitions, Phys. Lett. B 265, 258 (1991).

[5] D. Grasso and H. R. Rubinstein, Magnetic fields in the early universe, Phys. Rep. 348, 163 (2001).

[6] V. Skokov, A. Y. Illarionov, and V. Toneev, Estimate of the magnetic field strength in heavy-ion collisions, Int. J. Mod. Phys. A 24, 5925 (2009).

[7] V. Voronyuk, V. D. Toneev, W. Cassing, E. L. Bratkovskaya, V. P. Konchakovski, and S. A. Voloshin, (Electro-)magnetic field evolution in relativistic heavy-ion collisions, Phys. Rev. C 83, 054911 (2011).

[8] A. Bzdak and V. Skokov, Event-by-event fluctuations of magnetic and electric fields in heavy ion collisions, Phys. Lett. B 710, 171 (2012).

[9] W.-T. Deng and X.-G. Huang, Event-by-event generation of electromagnetic fields in heavy-ion collisions, Phys. Rev. C 85, 044907 (2012).

[10] K. Tuchin, Particle production in strong electromagnetic fields in relativistic heavy-ion collisions, Adv. High Energy Phys. 2013, 490495 (2013).

[11] C. S. Machado, F. S. Navarra, E. G. de Oliveira, J. Noronha, and M. Strickland, Heavy quarkonium production in a strong magnetic field, Phys. Rev. D 88, 034009 (2013).

[12] C.S. Machado, S. I. Finazzo, R. D. Matheus, and J. Noronha, Modification of the B meson mass in a magnetic field from QCD sum rules, Phys. Rev. D 89, 074027 (2014).

[13] J. Alford and M. Strickland, Charmonia and bottomonia in a magnetic field, Phys. Rev. D 88, 105017 (2013).

[14] D. Dudal and T. G. Mertens, Melting of charmonium in a magnetic field from an effective AdS/QCD model, Phys. Rev. D 91, 086002 (2015).

[15] S. Cho, K. Hattori, S. H. Lee, K. Morita, and S. Ozaki, Charmonium Spectroscopy in Strong Magnetic Fields by QCD Sum Rules: S-Wave Ground States, Phys. Rev. Lett. 113, 172301 (2014); QCD sum rules for magnetically induced mixing between $\eta_{c}$ and $J / \psi$, Phys. Rev. D 91, 045025 (2015).

[16] P. Filip, Heavy flavor mesons in strong magnetic fields, Proc. Sci., CPOD2013 (2013) 035.

[17] X. Guo, S. Shi, N. Xu, Z. Xu, and P. Zhuang, Magnetic field effect on charmonium production in high energy nuclear collisions, arXiv:1502.04407.

[18] E. Eichten, K. Gottfried, T. Kinoshita, J. B. Kogut, K. D. Lane, and T.M. Yan, Spectrum of Charmed QuarkAntiquark Bound States, Phys. Rev. Lett. 34, 369 (1975); 36, 1276(E) (1976).

[19] V. A. Miransky and I. A. Shovkovy, Magnetic catalysis and anisotropic confinement in QCD, Phys. Rev. D 66, 045006 (2002).

[20] M. A. Andreichikov, V. D. Orlovsky, and Y. A. Simonov, Asymptotic Freedom in Strong Magnetic Fields, Phys. Rev. Lett. 110, 162002 (2013).
[21] M. N. Chernodub, QCD string breaking in strong magnetic field, Mod. Phys. Lett. A 29, 1450162 (2014).

[22] R. Rougemont, R. Critelli, and J. Noronha, Anisotropic heavy quark potential in strongly-coupled $\mathcal{N}=4 \mathrm{SYM}$ in a magnetic field, Phys. Rev. D 91, 066001 (2015).

[23] E. J. Ferrer, V. de la Incera, and X. J. Wen, Quark antiscreening at strong magnetic field and inverse magnetic catalysis, Phys. Rev. D 91, 054006 (2015).

[24] H. Taya, Hadron masses in strong magnetic fields, Phys. Rev. D 92, 014038 (2015).

[25] Y. A. Simonov and M. A. Trusov, Confinement and $\alpha_{s}$ in a strong magnetic field, Phys. Lett. B 747, 48 (2015).

[26] E. J. Ferrer, E. S. Fradkin, and V. de la Incera, Effect of a background electric field of the Hagedorn temperature, Phys. Lett. B 248, 281 (1990).

[27] S. Ferrara and M. Porrati, String phase transitions in a strong magnetic field, Mod. Phys. Lett. A 08, 2497 (1993).

[28] E. J. Ferrer and V. de la Incera, Global symmetries of open strings in an electromagnetic background, Phys. Rev. D 49, 2926 (1994).

[29] E. J. Ferrer and V. de la Incera, Regge trajectories of the charged string in a magnetic background, Int. J. Mod. Phys. A 11, 3875 (1996).

[30] J. Ambjorn, Y. M. Makeenko, G. W. Semenoff, and R. J. Szabo, String theory in electromagnetic fields, J. High Energy Phys. 02 (2003) 026.

[31] C. Bonati, M. D'Elia, M. Mariti, M. Mesiti, F. Negro, and F. Sanfilippo, Anisotropy of the quark-antiquark potential in a magnetic field, Phys. Rev. D 89, 114502 (2014).

[32] G. S. Bali, QCD forces and heavy quark bound states, Phys. Rep. 343, 1 (2001).

[33] N. Brambilla, A. Pineda, J. Soto, and A. Vairo, Effective field theories for heavy quarkonium, Rev. Mod. Phys. 77, 1423 (2005).

[34] J. E. Avron, I. W. Herbst, and B. Simon, Separation of center of mass in homogeneous magnetic fields, Ann. Phys. (N.Y.) 114, 431 (1978).

[35] H. Herold, H. Ruder, and G. Wunner, The two-body problem in the presence of a homogeneous magnetic field, J. Phys. B 14, 751 (1981).

[36] T. Kawanai and S. Sasaki, Charmonium potential from full lattice QCD, Phys. Rev. D 85, 091503 (2012).

[37] J. Beringer et al. (Particle Data Group), Review of particle physics, Phys. Rev. D 86, 010001 (2012).

[38] L. D. Landau, E. M. Lifshits, and L.P. Pitaevskii, in Electrodynamics of Continuous Media, 2nd ed. (Pergamon Press, Oxford, 1984).

[39] E. Eichten and F. L. Feinberg, Spin-Dependent Forces in Heavy-Quark Systems, Phys. Rev. Lett. 43, 1205 (1979).

[40] E. Eichten and F. L. Feinberg, Spin-dependent forces in quantum chromodynamics, Phys. Rev. D 23, 2724 (1981).

[41] I. W. Sudiarta and D. J. W. Geldart, Solving the Schrödinger equation using the finite difference time domain method, J. Phys. A 40, 1885 (2007).

[42] M. Strickland and D. Yager-Elorriaga, A parallel algorithm for solving the $3 \mathrm{~d}$ Schrodinger equation, J. Comput. Phys. 229, 6015 (2010). 